\title{
Functionality Gaps in the Design of Learning Management Systems
}

\author{
Tallat Naz, Momeen Khan \\ Dept. of Computer Science \\ IIC University of Technology \\ Phnom Penh, Cambodia
}

\begin{abstract}
This research paper focuses on various gaps associated with the Learning Management System (LMS) and their remedies. LMS is a software application platform upon which multiple tasks related to online tutoring are created. For organizations, it's crucial that the risks associated with any automated process are kept as low as possible. This should also pertain to selecting the LMS platform for educating new professionals to the organization. To execute this, organizations, should carry out due research before incorporating any system as their primary LMS. Even though, they provide a lot of benefits for organizations integrating such platforms. Choosing faulty LMS for training recruits can lead to a variety of issues later on. Thus, it becomes essential to select the best LMS platform available in the market, and the one suits the organization's needs. The work proposed in this paper is listing together a number of problems that exists in any given LMS framework and trying to alter them according to the needs of the organization so that they provide a feasible solution and deliver a better guidance to the recruits.
\end{abstract}

Keywords-Learning Management System (LMS); shortcomings of LMS; functional gaps in LMS; LMS design issues; remedies for gaps in design of LMS

\section{INTRODUCTION}

Learning has always been an essential pursuit for humankind since ancient times. With the advent of the internet, the quantity and quality of information available to a person now have increased exponentially. For the traditional learning method, the Internet has allowed to be side-lined gradually and is replaced by electronic learning methods [1]. These all electronic-learning methods are collectively called elearning. It is the broad term used to define any formal teaching done with the help of electronic devices. Internet and computers form essential components of the e-learning method, but is not limited to these. E-learning includes everything from early implementations such as pre-recorded CD \& DVD, to the latest innovation such as live, interactive classes, where the teacher and students can interact even when they are on the other side of the planet. E-learning offers a variety of advantages over the conventional teaching methods such as cost-saving measures and ability to learn around the clock. With the advancement of mobile technology, the new concept of m-learning has also developed, that is e-learning done through mobile devices [2]. It is estimated that the percentage of using e-learning methods is steadily rising in the last few years. According to statistics, nearly $99 \%$ of higher education institutions currently adopt and run LMS platforms
[3]. According to an Educause Centre for Analysis and Research (ECAR) survey, 86\% of teachers use LMS and out of which $56 \%$ use it on a daily basis [4]. $83 \%$ of students use LMS in at least one course with $56 \%$ using it in most or all courses [5]. This demonstrates that teachers and students value the LMS as an enhancement to their educational experience.

In this paper, we focus on the difficulties that are encountered by the organizations that rely on LMS platforms for their day-to-day usage. These organizations can be educational or corporate. A number of strategies are also provided for correcting these difficulties. These are the problems that can have undesired results for the organizations and can often result in the delay of the training or educational process.

This paper helps in contribution towards the ever-present problems that many organizations face, i.e. the training of recruits. For this purpose, implementing an LMS framework is convenient and practical. Thus, it becomes crucial that the LMS selected can fulfill all the necessary requirements that an organization can have and manage them well. Some of the common functionalities of modern LMS are shown in the fig. 1.

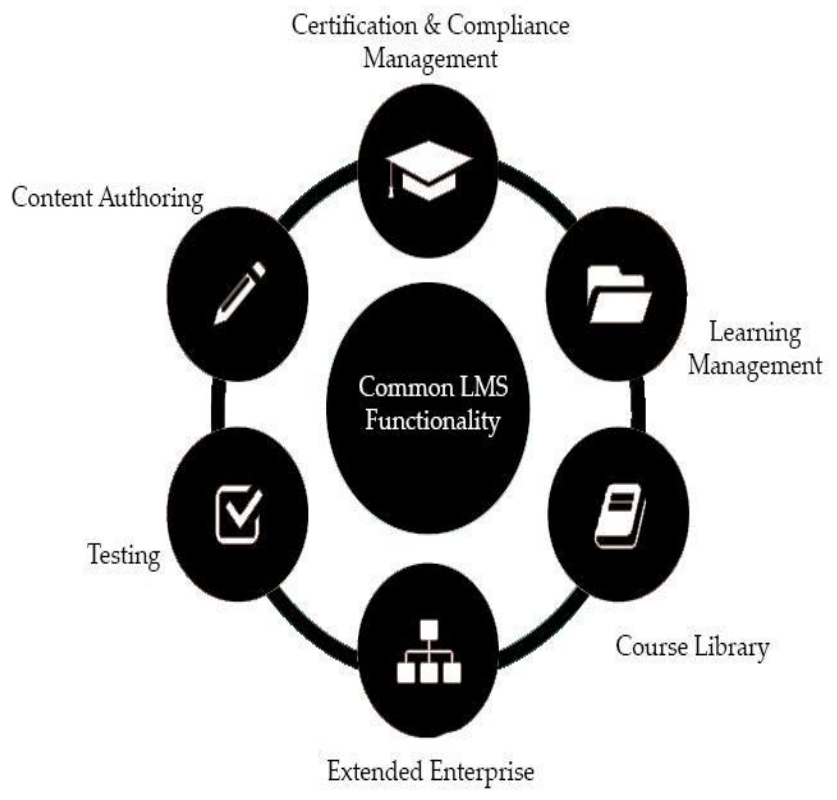

Fig. 1. Functionalities Common to All Modern Lms. 


\section{LMS AND ITS MODELS}

Learning Management System (LMS) is an online platform that allows a user to carry out many tasks related to e-learning. LMS can be understood as the mechanism that powers e-learning. The functions of an LMS can range from essential services such as providing educational content to tracking a user's progress throughout the course's timeframe and conducting a regular assessment. LMS can also perform other processes such as chat boxes for users, periodic tests and other necessary tasks related to teaching. All organizations that have to educate recruits, either students or professional, can benefit from LMS. But deciding the correct LMS to incorporate is the major hurdle organizations have to go through. A variety of options are available that differ from each other in functionality and expenses incurred[6]. Two different models of LMS are explained below.

\section{A. Massive Open Online Courses (MOOC)}

MOOCs are sites that furnish an online course with a many choices. These choices are free and open, provide generally shared educational modules, and convey open-ended results. MOOCs coordinate social networking, data that are available online open on the web, and are advanced by top professionals in the field of study[7]. Most strikingly, MOOCs expand on the engagement of researchers who self-compose their support as per learning objectives, earlier information and abilities, and comparative interests.

\section{B. Software based LMS}

The same concept of MOOC when performed through the use of software is said LMS software. There are two types of LMS software that is widely available, each with its advantages and disadvantages.

1) Open Source LMS: These are software that is free-touse for everyone and organizations can modify upon the existing platform provided by the developer community. The most popular example of this category is Moodle [8]. They offer free of cost LMS solution but come with many hidden costs such as hosting fees, extra storage space, and more tech support [9].

2) Proprietary LMS: Proprietary LMS platform is those that are developed by for-profit organizations. Blackboard is an example of the patented LMS with the maximum market share [10]. These are comparatively more secure and reliable than open-source LMS. They also enable the creation of training courses. But the higher cost associated with might keep smaller companies at bay.

\section{ANALYSIS OF VARIOUS COMPETITORS OF LMS PLATFORM}

The LMS platform market has many competitors that have cemented their place amongst the consumers. Some of the top systems consist of both open-source and proprietary platforms. A quick analysis of the market share provides us with some important information.

\section{A. Market Share in American Higher Education Institutions}

The market share of all the top LMS competitors which are utilized in institutes with more than 2000 students is analyzed below. The two systems that are at the top, that is, Blackboard \& Canvas are both proprietary LMS. The market share of Moodle has remained stable over the last few years but has fallen in terms of total usage. When comparing with institutes with less than 2000 students, the trends remain the same and Blackboard is again the majority holder, as shown in the fig. 2.

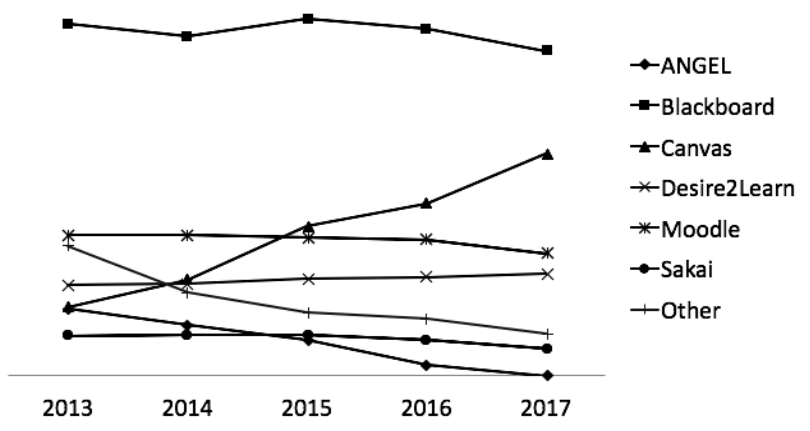

Fig. 2. Market Share in US [11].

\section{B. Global Scenario of LMS}

When observed in the global standings, we get approximately the results with Moodle jumping to second position. Canvas is nearly non-existent in other top markets for LMS, such as UK \& Canada as shown in fig. 3.

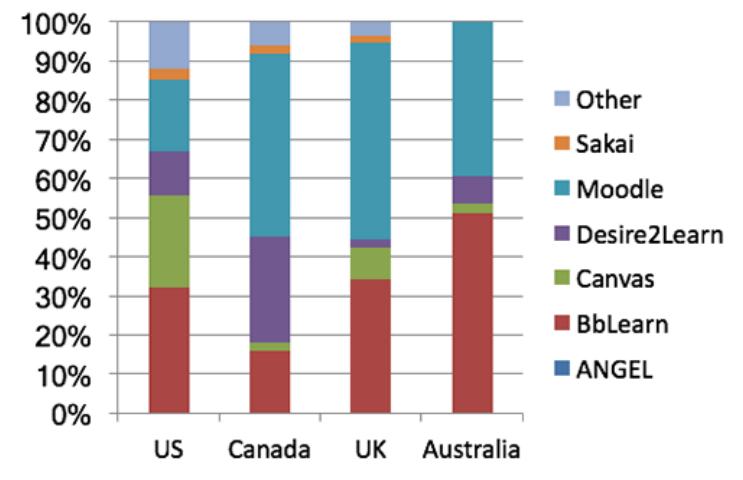

Fig. 3. Holdings in 4 Biggest Markets of LMS.

\section{SHORTCOMINGS OF LMS}

Most organizations have to adhere to the standards approved by the regulatory bodies of EU and USA. As LMS is the integral part of the development and learning process, they have to keep the vigilance on the LMS they operate on. Due to the several risks that are associated with using an LMS with any flaw, it is imperative for the organizations to select the best product that fulfils all their requirements. 
The various potential gaps of the Learning Management System are addressed below:

\section{A. Lack of Version Control}

The first gap of LMS is the lack of version control for the training content, which can result in two major issues. The first and foremost is the increased rate of human error. Because of the higher human error rate, the collected content can be deemed unreliable. This can increase the risk of wrong information being transverse. The other one is an absence of automation for version training and reviewing. This can lead to considerable in-efficiencies for the administrative department. There are many Corrective Action/Preventive Action (CAPA) directives which will be breached due to this gap.

\section{B. The Absence of Computerized Electronic Markings or e- Signatures}

The absence of computerized electronic markings or esignatures builds a level of deficient in preparing results and record maintenance, which leads to inconsistencies in the documentation process. E-signatures require extra managerial action as they don't automatically add to every one of the courses associated with the training program and as a result, can confuse the students.

\section{Deficiency of Audit Trail}

The third shortcoming is the deficiency of audit trail data on curriculum profiles and learner's training items. Finite data on what was changed and who rolled out the improvement, failure to hold the information for the required time and day, and the absence of date and time-stamp capacity. This hinders the preparation program control and observing, and lessens the impact of the ability to build up an extensive, end-to-end errand review trail. The downside of an LMS to record both old and new execution of benchmarks makes the framework inconsistent for Code of Federal Regulations (CFR). The need to physically screen which is contributing the framework changes, the date and time the progressions were made, and who rolled out the improvements, can likewise cause the LMS not agreeable to CFR mandates.

\section{Managerial and Administration Consent}

Another gap of LMS is that all managerial and administration consent should be performed physically, outside the LMS if the preparation design endorsement processibility is inadequate. Administration unequipped for surveying singular preparing plans against worker sets of expectations with a specific end goal to guarantee precision when enlisted and refreshed when there is an adjustment in part. With expanding occurrence of difficult work fundamental to make up for the absence of electronic forms, the margin for the human mistake would likewise increment. Therefore, extra work-force must be sent to carry out a manual audit of the preparation program.

\section{E. Insufficient "Audit-Ready" Reports}

Insufficient "audit-ready" reports for managers and administrators indicate the real-time qualification's status of learners. Administrators and managers must get immediate notification and access to the qualification and the compliance level of their employees. These records also need to be presentable during an audit. They must have all necessary data so that it seems it is represented from a trustworthy and accurate source.

\section{F. No Automated Role-Based Training Management Features}

As the highlights of LMS and coordinating usefulness that represents the part based preparing process are inadequate, the staff engaged with the quality framework can't be assembled into automated parts relying on their activity capacities. Development, evaluation, approval, and assignment of individual training plans have to be done manually when there are no automated role-based training management features. This raises the issue of human mistakes and can give the supervisors the impression that the education plans are assigned inconsistently. It can indicate that the employees are not adequately trained before performing the activity.

\section{G. Incapable of Tracking the Training Equivalencies}

Administrators and managers can't unmistakably assess, set down, allot and report comparable preparing performed in a few preparing modalities/strategies.

The current LMS's are unequipped for following the preparation equivalencies between different learning exercises. The functionality accessible to address those equivalencies is constrained, and just a similar preparing technique/sort can be made identical. For example, English and German adaptations of related material can be made similar, yet slightly different types of learning sources are past this parameter. Along these lines, a read and sign preparing on a SOP or an educator drove preparing on a SOP isn't created as, or seen as a comparable in the LMS.

\section{ADDRESSING THE GAPS}

The gaps mentioned above of the LMS platform can be corrected by carrying out the following processes.

\section{A. Acquiring Adaptation Control Capacities}

Acquiring adaptation control capacities can dispose of manual compromises for a developing volume of different material forms. It ensures the organization against the issuance of warning letters, and other review remediation orders. It improves the working efficiency of both the system and quality organization.

\section{B. Electronic Marking and Records Consistent Or E- Signature Production}

Electronic marking and records consistent with stringent necessities of FDA, CFR and EU Annex will permit that the esignature must be produced toward the fruition of preparation. It must keep up security and review trails to guarantee it is an immaculate record. It must produce the approval of frameworks to guarantee unwavering quality, exactness, the capacity to observe adjusted or invalid records, and predictable, planned execution. It must generate the time of completion and exact duplicates of documents in both electronic and comprehensible frame appropriate for assessment, examination, and replicating by the administrative office. 


\section{Date and Time-Stamped Review Trails}

The time and date of operator activities and operations that create, change, or delete electronic records can be freely recorded by secure, computer produced, date and timestamped review trails..

\section{Intermittent, Computerized Surveys of Preparing Plan Usefulness}

Limit and minimize the reliance on manual and administrative actions. It can be adjusted to coordinate the interior preparing record maintenance disciplines that will boost responsibility, oversight, and control of preparing plan surveys.

\section{E. Automated Reports Preparations}

Automated reports preparations and notices progressively that can detail the capability and consistency status of students. It informs both framework heads and directors using email when a worker's task is past due. It offers current access to preparing records continuously. It produces "review prepared" reports in pdf format and arranges them according to date/time stamps, report criteria, page numbers, and so forth.

\section{F. Full Automation of the Critical Preparing Plans}

Full automation of the critical preparing plans from representative on-boarding to progressing capability for those staff engaged with GxP activities. It guarantees making of steady preparing records for similar courses, and reliable reporting and tracking. It empowers the staff to satisfy the preparation objective necessary to get to access system or conduct operations and guarantees the opportune individuals are prepared at the ideal time on the correct material.

\section{G. Following of Training Equivalency between Different Learning Sources}

Limits the human mistake caused by physically taking care of representative preparing fruitions and oversight of records. A top-tier report for each kind of preparing can be united by system managers, making it less demanding to run reports and react to internal info demands.

\section{DISCUSSION}

Observing the above-mentioned risks, it is imperative that the LMS selected for the usage in organizations are the best product possible and a product that meets all the requirements. Many of the top products available in the market have gained market penetration which hinders the decision making of the organizations. To avoid that, organizations should survey all the available options. Many new products are streamlined for particular tasks which are better suited for the organization. Any error on the part of LMS can have significant effects on the working of these organizations. To avoid these mistakes, it is essential that the organizations take in mind all the different options available for the LMS selection and choose the best option possible.

\section{CONCLUSION}

Learning management system is playing a vital role in the learning and development process of any organization. If applied accurately, the rectifications offered by this research paper will help the organizations to respond to evolving requirements that comes with training new professionals. It is of utmost importance that the recruits are trained as best as possible. The LMS platform created after correcting all the variety of issues mentioned above can help an organization to provide a well-organized experience.

The future application of the proposed drawbacks and their improvement can help the organizations to make an informed decision about the various drawbacks present in LMS platforms and have to be avoided. There are a number of opportunities available for future development that can be pursued by making this particular study as the basis for advance research.

\section{ACKNOWLEDGMENT}

We would like to express our gratitude to Prof. Dr. Chhuon Chanthan and IIC University of Technology, Phnom Penh, Cambodia for providing us good support to conduct this work.

\section{REFERENCES}

[1] Andersson, A., (2008). Seven Major Challenges for e-learning in Developing Countries:Case Study eBIT, Sri Lanka, International Journal of Education and Development using ICT, Vol 4, Issue 3

[2] Hunsinger, J. (2005). How to determine your readiness for mobile elearning.Information policy. http://ipolicy.typepad.com/informationpolicy/2005/04/how_to_determin. html

[3] Dahlstrom, E., Brooks, D. C., \& Bichsel, J. (2014). The current ecosystem of learning management systems in higher education: Student, faculty, and IT perspectives. Research report. Louisville, CO: ECAR. https://net.educause.edu/ir/library/pdf/ers1414.pdf

[4] Berking, P., \& Gallagher, S., (2016). Choosing a Learning Management System (Ver. 7.0). Advanced Distributed Learning (ADL) CoLaboratories, 14, 40-62.

[5] Brown, M., Dehoney, J., \& Millichap, N. (2015). The Next Generation Digital Learning Environment. A Report on Research. Educause Learning Initiative paper. Retrieved from https://net.educause.edu/ir/library/pdf/eli3035.pdf

[6] Yuan, L. \& Powell, S. (2013). MOOC and Open Education: Implications for Higher Education. CETIS: Centre for Educational Technology, Interoperability and Standards, White Paper, WP03, http://publications.cetis.org.uk/2013/667

[7] Diver, P., \& Martinez, I. (2015). MOOCs as a massive research laboratory: Opportunities and challenges. Distance Education, 36(1), 525. http://dx.doi.org/10.1080/01587919.2015.1019968

[8] Feller, J., \& Fitzgerald, B. (2000). A framework analysis of the open source software development paradigm. In Proceedings of $21 \mathrm{st}$ International Conference on Information Systems (pp. 58-69). Australia: Brisbane.

[9] Aydin, C. C., \& Tirkes, G., 2010: Open source learning management systems in e-learning and Moodle. InEducation Engineering (EDUCON), 2010 IEEE (pp. 593-600).

[10] Pankaja, N., \& Mukund Raj, P. K. (2013). Proprietary software versus open source software for education. American Journal of Engineering Research, 2(7), 124-130.

[11] edutechnica.com 